after correcting for head size and sex. (Thompson DK, Wood SJ, Doyle LW, et al. Neonate hippocampal volumes: prematurity, perinatal predictors, and 2-year outcome. Ann Neurol May 2008;63:642-651). (Resapond: Deanne K Thompson, BSc(Hons), Howard Florey Institute, Level 2, Alan Gilbert Building, 161 Barry Street, Carlton South, VIC 3053, Australia).

COMMENT. The hippocampus, part of the limbic lobe, originally linked mainly with olfactory function, is now considered important in memory, spatial function, and cognition. Hippocampal volume reduction reported in children with chromosome 22q11.2 deletion syndrome is correlated with severity of cognitive impairment. (Deboer T et al. Behav Brain Funct 2007;3:54). Severe memory impairment is reported in a 5-year-old child with marked hippocampal atrophy after prolonged status epilepticus. (Jambaque I et al. Dev Med Child Neurol 2007;49:398-399). The authors of the above report in infants recommend further MRI studies in older children to determine the role of the hippocampus in the high rate of cognitive impairment in preterm infants tested at a later age. They advocate interventions to decrease white matter damage and overuse of postnatal steroids and indomethacin in preterm infants, factors linked to smaller hippocampal volume.

\title{
VERY PRETERM BIRTH, CEREBELLAR DEVELOPMENT AND NEUROPSYCHOLOGICAL OUTCOME IN ADOLESCENCE
}

Cerebellar volumes were measured on structural MRI at adolescence and adulthood in 65 preterm individuals (born before 33 weeks' gestation), and a term-born comparison group, in a study at King's College, Great Ormond Street Hospital, and University College, London; and Seoul National University College of Medicine, Korea. Cerebellar volumes in late adolescence and adulthood (mean age $18.6 ; \mathrm{SD}=1.02$ ) were $3.11 \%$ and significantly smaller than measurements during early adolescence (mean age 15 years; $\mathrm{SD}=1.43$ ) in the preterm group $(\mathrm{P}=0.000)$, whereas cerebellar volumes increased $0.44 \%$, but did not change significantly with age in the control group $(\mathrm{P}=0.612)$. The changes in cerebellar volume correlated with tests of behavior and cognitive function. High General Health Questionnaire (GHQ)-12 scores, a self-reporting wellbeing test (eg feeling worthless, poor concentration), indicative of increased risk of mental health problems, correlated with reduction in cerebellar volume during late adolescence and young adulthood. Cerebellar volume correlated positively with full scale, verbal and performance IQ in early adolescence in the very preterm group but not the term-born group. Correlations with IQ were not maintained after controlling for white matter volume. (Parker J, Mitchell A, Kalpakidou A, et al. Cerebellar growth and behavioural \& neuropsychological outcome in preterm adolescents. Brain May 2008;131:1344-1351). (Respond: Matthew Allin, Kung's College London, Division of Psychological Medicine and Psychiatry, Institute of Psychiatry, London, UK. E-mail: matthew.allin@iop.kcl.ac.uk)

COMMENT. A decrease in cerebellar volume occurring between mean age 15 years and 18.6 years in very preterm individuals is correlated with impaired feelings of wellbeing, but a correlation with IQ deficits is not significant when controlled for white matter volume. These findings corroborate previous reports of cerebellar involvement in cognitive and neurobehavioral disorders. (Murakami JW et al. Arch Neurol 1989;46:689-694). 